\title{
Urinary excretion of dithiocarbamates and self-reported Cruciferous vegetable intake: application of the 'method of triads' to a food-specific biomarker
}

\author{
Jay H Fowke ${ }^{1, *}$, James R Hebert ${ }^{2}$ and Jed W Fahey ${ }^{3}$ \\ 'Vanderbilt-Ingram Cancer Center, Vanderbilt Center for Health Services Research, and Department of General and \\ Internal Medicine, Vanderbilt University Medical Center, Vanderbilt University, 6110 Medical Center East, Nashville, \\ TN 37232-8300, USA: ${ }^{2}$ Department of Epidemiology and Biostatistics and the Nutrition Research Center, Norman J \\ Arnold School of Public Health, University of South Carolina, Columbia, SC, USA: ${ }^{3}$ Department of Pharmacology \\ and Molecular Sciences, School of Medicine, and Center for Human Nutrition, Bloomberg School of Public Health, \\ Johns Hopkins University, Baltimore, MD, USA
}

Submitted 27 August 2001: Accepted 28 February 2002

\begin{abstract}
Objective: Greater intake of Cruciferous vegetables (e.g. broccoli) may prevent cancer at several sites. Urinary excretion of isothiocyanate conjugates (dithiocarbamates, DTC) provides a specific biomarker of Cruciferous vegetable consumption suitable for epidemiological investigations. However, no gold-standard referent is available for evaluating urinary DTC levels as an estimator of Cruciferous vegetable consumption. We compared urinary DTC levels to intake as measured by two selfreported dietary assessment techniques.

Design: Cruciferous vegetable consumption was measured before and after a behavioural dietary intervention using multiple 24-hour recalls (24HR), a foodcounting questionnaire (VFQ) and urinary DTC excretion levels. Analysis included a structural equation approach (Method of Triads) combining these three assessment techniques to estimate the relationship between DTC level and the study population's 'true' Cruciferous vegetable intake.

Setting: The intervention curriculum assisted participants in consuming about 2 servings per day for a 6-week period. Participants attended four classes emphasising problem-solving skills, dietary counselling and vegetable preparation skills. There were no dietary restrictions.

Subjects: Thirty-three healthy, free-living, post-menopausal women.

Results: Although few participants reported Cruciferae consumption prior to the intervention, 30 participants reported Cruciferae consumption after the intervention (Post-intervention). Urinary DTC levels were correlated with estimated intake derived from either the 24HR ( $r=0.57$; 95\% confidence interval (95\% CI) $0.28,0.76$ ) or VFQ ( $r=0.49 ; 95 \%$ CI $0.17,0.71$ ). The validity coefficient (Method of Triads) between urinary DTC excretion and an index of true Cruciferous intake was stronger than the Pearson correlation $\left(r_{\mathrm{v}}=0.65 ; 95 \%\right.$ CI $\left.0.35,0.90\right)$, and comparable to estimates derived from the 24HR $\left(r_{\mathrm{v}}=0.82 ; 95 \%\right.$ CI $\left.0.65,1.00\right)$ or VFQ $\left(r_{\mathrm{v}}=0.76 ; 95 \%\right.$ CI 0.47 , $0.92)$ method. These associations were not affected by adjustment for body mass index, energy intake, or social approval or desirability response sets.

Conclusions: Food-frequency questionnaires (FFQ) suitable for large epidemiological studies may not be designed to measure all Cruciferae, and cannot capture exposure to phytochemicals derived from those vegetables. Urinary DTC measurement was significantly correlated with Cruciferae intake derived from two dietary assessment approaches, and urinary DTC levels could supplement traditional FFQ data by providing an index of recent Cruciferous vegetable intake not susceptible to reporting biases.
\end{abstract}

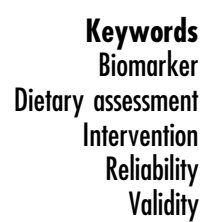

The consumption of Cruciferous vegetables, including broccoli and cabbage, results in the enzymatic conversion of the plant glucosinolates to metabolites that are predominantly isothiocyanates (ITC). These ITC are metabolised in the body to form glutathione conjugates which then undergo further enzymatic modification. These conjugates are collectively known as dithiocarbamates (DTC). Cruciferous vegetables and ITC are potent 
inducers of Phase 2 enzymes such as glutathione $S$ transferase (GST) and facilitate the metabolism and detoxification of xenobiotics, some of which are carcinogens. Recent epidemiological studies suggest that the addition of Cruciferae to the habitual diet may reduce cancer risk ${ }^{1-3}$. However, it has been difficult to isolate the effects of Cruciferae from other diet- or health-related behaviours. Food-frequency questionnaires (FFQs) employed in epidemiological studies are limited in providing estimates of specific food types. In addition to its susceptibility to reporting biases ${ }^{4,5}$, the FFQ queries a limited range of food items (thus omitting some sources of ITC), is limited to a rank ordering of vegetable intake across subjects, and is unable to capture differences in phytochemical exposure due to the specific cultivars of particular vegetables consumed (because the FFQ aggregates across categories), cooking practices, or even actions as apparently inconsequential as the way in which food is chewed ${ }^{6}$.

Dietary biomarkers provide an objective exposure measure for a particular phytochemical, and are unaffected by potential reporting biases commonly found in FFQ evaluation ${ }^{7}$. A 'cyclocondensation' assay has been developed by exploiting a chemical reaction in which both free ITC and their glutathione, cysteinylglycine, cysteine and $\mathrm{N}$-acetylcysteine conjugates (collectively termed dithiocarbamates; DTC) react with 2-benzenedithiol to create reaction products with highly favourable properties for spectroscopic detection, separation from a body fluid matrix and sensitive quantification ${ }^{8,9}$. In clinical in-patient studies and observational studies, urinary DTC levels increase in a dose-response manner following the consumption of several common Cruciferous vegetables $^{10,11}$. The response is specific to the vegetables consumed and individual GST genotype ${ }^{6,8,10,11}$, and urinary DTC excretion has been significantly associated with reduced lung cancer risk $^{12}$. The urinary DTC biomarker thus provides an opportunity to focus on Cruciferae-disease investigations with specificity that was hitherto unobtainable.

The complete evaluation of urinary DTC levels as a biomarker of Cruciferous vegetable intake should include male and female subjects across race and age groups. We recently conducted a dietary intervention to investigate obstacles to dietary change and the relationship between Cruciferous vegetable consumption and markers of cancer risk among post-menopausal women living in the United States $^{13}$. We measured the DTC concentration in 24-hour urine samples collected from each participant before and after the dietary intervention. Adherence to the dietary intervention was measured by repeated 24-hour dietary recalls (24HR), a reference dietary assessment approach, and with a vegetable and fruit questionnaire (VFQ) containing all common Cruciferous vegetables. With these three separate assessment methods (biomarker, 24HR, VFQ), we have attempted herein to expand the analysis beyond correlation and regression by including a structural equation approach (the Method of Triads $^{7}$ ). This approach simultaneously combines data from the three methods to provide a coefficient between each dietary assessment technique and the true (but unknown) level of Cruciferous vegetable consumption.

\section{Materials and methods}

\section{Subjects}

Participants were healthy post-menopausal women. They did not use tobacco or exogenous hormones, and had no medical restrictions on their diet. Each participant had not had a menstrual cycle during the prior 12 months, and did have a negative screening mammogram within the past year.

\section{Data collection}

The single-armed intervention facilitated the consumption of Cruciferous vegetables among 34 healthy postmenopausal women. The dietary intervention consisted of four classes over a four-week period. These classes provided an opportunity to instruct study participants about the theorised health benefits of Cruciferae consumption, and to monitor them for any adverse reactions. The dietary goal of the intervention was to increase the Cruciferous vegetable consumption of freeliving women to levels consistent with the intake observed in certain Asian regions (i.e. about one or two servings per day) ${ }^{14}$.

Instructions for collecting a 24-hour urine sample were provided to each participant in both written and oral formats, and $2 \mathrm{~g}$ of ascorbic acid was added to each opaque urine collection container. Each subject provided a 24-hour urine sample during the week prior to the intervention (Baseline) and approximately two weeks following the conclusion of the intervention (Postintervention). Participants delivered the urine sample to research personnel on the day that collection was completed, urine volumes were immediately measured, and aliquots were stored at $-80^{\circ} \mathrm{C}$. Each participant's weight was measured at each of these study phases, and height was measured at baseline.

\section{VFQ description}

The VFQ (see Appendix) is a self-administered 37-item questionnaire that queries the consumption of specific functional foods and fruits during the previous seven days. As such, it attempts to obtain actual counts of exposure and not frequency categories, much along the same lines as the 7-day dietary recall we developed for assessing change in dietary fat ${ }^{15}$. Vegetables and fruits were identified from the research literature as being sources of phytochemicals or nutrients of interest for further cancer prevention research. Cruciferous vegetables on the VFQ included broccoli, broccoli sprouts, cabbage, Brussels 
sprouts, cauliflower, Chinese cabbage, collards/Swiss chard/kohlrabi, mustard greens/turnip greens, rutabaga/turnips and kale. A standard portion size is provided for each food on the VFQ. Participants completed the VFQ by indicating their typical portion size as compared to a referent portion, usually a volume such as one-half cup. Participants then indicated the number of times that this portion size was consumed during the prior 7 days. Portions consumed were converted to grams, based on conversion factors derived from the Nutrition Data System (version 2.92; University of Minnesota, Minneapolis, MN), and these amounts were divided by 7 to produce an average daily intake. Conversions from portions of Cruciferae to weights of Cruciferae were based upon the cooked food weight, since in the United States, most Cruciferous vegetables on the VFQ are usually consumed after cooking. The VFQ does not query food preparation methods. The VFQ was completed at the end of the week in which a urine sample was provided. Thirty-four women completed the entire VFQ at Post-intervention study phases; however, the VFQ was only administered to 28 women at Baseline due to a delay in editing the questionnaire. The VFQ questionnaire was incomplete for one study participant, and therefore the analysis is restricted to data from 27 participants at Baseline and 33 participants at Post-intervention. Only the Cruciferous vegetable section of the VFQ was analysed for the purposes of this study.

\section{Coding of the 24-bour recalls}

The 24-hour recall interview (24HR) was used as a referent estimate of dietary intake. Total variability in nutrient values from repeated $24 \mathrm{HR}$ have been shown to be lower than food diary nutrient scores, and the $24 \mathrm{HR}$ is less likely to produce changes in the participants' food intakes than the diary ${ }^{16}$. Participants completed three $24 \mathrm{HR}$ within the same weeks that 24-hour urine samples were collected. Two weekdays and one weekend day were randomly selected within each of these weeks, and trained dietitians conducted the telephone interviews. In an effort to improve the accuracy of portion size estimation, prior to the 24HR dietary assessment each participant was mailed two-dimensional pictures illustrating the usual portion sizes of several common foods.

Nutrient values were calculated using the Nutritional Data System (version 2.92; University of Minnesota, Minneapolis, MN). Each $24 \mathrm{HR}$ was scanned for food identification codes indicating a Cruciferous vegetable. The amounts of each Cruciferous vegetable reported on each day were combined across days within each study phase, providing a single estimate of the participants' daily Cruciferous vegetable consumption during each study phase.

\section{Ditbiocarbamate laboratory analysis}

Urinary DTC levels have been found to be a sensitive biochemical marker for recent Cruciferous vegetable consumption $^{6,8,10}$, representing a unique indicator of exposure among food groups. The laboratory analysis was performed as described by Zhang and colleagues ${ }^{9}$, and adapted for urinary DTC determination ${ }^{6,10}$. In brief, samples $(1.5 \mathrm{ml})$ from each urine collection were thawed and centrifuged $\left(200 \mathrm{~g}\right.$ for $5 \mathrm{~min}$ at $4^{\circ} \mathrm{C}$ ). Urine (200 or $500 \mu \mathrm{l})$ and enough water to total $500 \mu \mathrm{l}, 0.5 \mathrm{ml}$ of $500 \mathrm{mM}$ sodium borate buffer ( $\mathrm{pH} 9.25$ ) and $1.0 \mathrm{ml}$ of $40 \mathrm{mM} \mathrm{1,2-}$ benzenedithiol in methanol were added to $4 \mathrm{ml}$ vials. The vials were flushed with $\mathrm{N}_{2}$ gas, sealed with Teflon-lined septa and incubated for 2 hours at $65^{\circ} \mathrm{C}$. Samples were then cooled to room temperature, briefly centrifuged ( $350 \mathrm{~g}$ for $5 \mathrm{~min}$ ), and loaded into an autosampler for highperformance liquid chromatography (HPLC). All chromatographic conditions, standard curves, assay reproducibility, linearity of response and storage characteristics of urine samples were as detailed in Shapiro et $a l^{10}$. Although more recent methodological improvements have improved the sensitivity by depleting proteins in the sample and concentrating the cyclocondensation product (1,3-benzenedithiole-2-thione) $)^{8}$, this method as used was sensitive to $15 \mathrm{pmol}$ conjugated 1,3-benzodithiole-2-thione in $50 \mu \mathrm{l}$ of urine. Concentrations below $15 \mathrm{pmol} / 50 \mu \mathrm{l}$ were considered non-detectable, and urinary DTC concentration was multiplied by the volume of urine collected during the 24-hour period $(\mathrm{ml})$ and is presented as $\mu \mathrm{mol}$ daily DTC excretion.

\section{Social desirability and social approval}

A person with a high social desirability score is one who is highly aware of society's perceptions of appropriate behaviour and evinces a defensive tendency to be viewed in a socially acceptable way in situations in which she perceives she is being tested. A person with a high social approval score is one who seeks approval in a testing situation. Dietary reporting has previously been associated with an individual's sensitivity to the social pressures influencing self-report of diet'. Both 'social desirability'17 and 'social approval'18 were measured in each participant to evaluate the impact of these psychosocial constructs on the relationship between the VFQ, DTC biomarker and $24 \mathrm{HR}$.

\section{Statistical analysis}

Participants completed the VFQ at the end of the 7-day period in which both the $24 \mathrm{HR}$ interviews were performed and the 24-hour urine samples were collected. Parallel analyses were conducted using data from both the Baseline and the Post-intervention study phases in order to compare the relationships between urinary DTC and VFQ or $24 \mathrm{HR}$ measures within the same individuals but at different levels of Cruciferous vegetable consumption (on the correct assumption that the intervention had resulted in increased intake of these vegetables). The DTC data were skewed right and these data were natural log (base e) transformed to reduce heteroscedasticity in the regression 
analysis. Scatter plots and least-squares linear regression were used to investigate the relationship between VFQ, 24HR and urinary DTC data.

Pearson correlation coefficients described the strength of the linear association between the VFQ, 24HR and DTC data. Ninety-five per cent confidence intervals (95\% CIs) for each correlation coefficient were calculated using a Fisher's $\mathrm{Z}$ transformation ${ }^{19}$. The validity coefficient mathematically estimating the association between either urinary DTC levels, VFQ estimates or the 24HR estimates and the latent true intake of Cruciferae has been developed by Kaaks and colleagues, using a structural equation approach labelled the Method of Triads ${ }^{7,20}$. This approach assumes an independent error structure from at least one measurement, normality, and a linear association between each measurement and the true (unknown) value. While a correlation coefficient is restricted to the range $(-1,1)$, it is mathematically possible for a validity coefficient to exceed these ranges. This may occur due to chance alone or because an assumption was violated; a mathematical result of this kind is referred to as a Heywood case $^{7}$. The final calculation for the validity coefficient for each measure and the unknown true intake of Cruciferous vegetables is determined by the square root of a product function of the Pearson correlation coefficients, as follows:

$$
\begin{aligned}
& r_{\mathrm{VFQ} \times \mathrm{t}}=\sqrt{\left(r_{\mathrm{DTC} \times \mathrm{VFQ}} \times r_{\mathrm{VFQ} \times 24 \mathrm{HR}}\right) / r_{24 \mathrm{HR} \times \mathrm{DTC}},} \\
& r_{24 \mathrm{HR} \times \mathrm{t}}=\sqrt{\left(r_{\mathrm{DTC} \times 24 \mathrm{HR}} \times r_{\mathrm{VFQ} \times 24 \mathrm{HR}}\right) / r_{\mathrm{VFQ} \times \mathrm{DTC}}}
\end{aligned}
$$

and

$$
r_{\mathrm{DCT} \times \mathrm{t}}=\sqrt{\left(r_{\mathrm{DTC} \times \mathrm{VFQ}} \times r_{\mathrm{DTC} \times 24 \mathrm{HR}}\right) / r_{\mathrm{VFQ} \times 24 \mathrm{HR}}} .
$$

Confidence intervals for each validity coefficient were estimated using 1000 bootstrap samples of the same size as the number of subjects in the dataset. Partial correlation coefficients were calculated to produce adjusted validity coefficients with regard to age, body mass index (BMI) (weight $(\mathrm{kg}) /$ height $(\mathrm{m})^{2}$ ), energy intake $\left(\mathrm{kcalday}^{-1}\right)$, social approval or social desirability score.

\section{Results}

The 33 post-menopausal women who participated in this study had an average age of 62 years (range: 49-77 years), $73 \%$ were married, and $82 \%$ had at least a high school education.

As estimated by $24 \mathrm{HR}$, Cruciferous vegetable consumption at Post-intervention averaged approximately $140 \mathrm{~g} \mathrm{day}^{-1}$, between 4 and 10 times higher than at Baseline (Table 1). Only three participants failed to consume these vegetables following the intervention. There was a significant linear component to the relationships between each measurement of Cruciferae intake (Fig. 1). Two subjects had urinary DTC excretion
Table 1 Vegetable consumption $\left(\mathrm{g} \mathrm{day}^{-1}\right)$ and dithiocarbamate excretion $\left(\mu \mathrm{mol} \mathrm{day}^{-1}\right)$

\begin{tabular}{lrrrrr}
\hline & \multicolumn{2}{c}{$\begin{array}{c}\text { Baseline } \\
(n=27)\end{array}$} & & \multicolumn{2}{c}{$\begin{array}{c}\text { Post-intervention } \\
(n=33)\end{array}$} \\
\cline { 2 - 3 } \cline { 6 - 6 } & Mean & SD & & Mean & SD \\
\hline DTC & 5.3 & 9.9 & & 20.4 & 22.3 \\
VFQ & 23.6 & 20.7 & & 190.1 & 110.6 \\
24HR & 14.5 & 20.7 & & 140.4 & 89.1 \\
\hline
\end{tabular}

SD - standard deviation; DTC - urinary dithiocarbamate excretion (not transformed); VFQ - vegetable and fruit questionnaire; $24 \mathrm{HR}-24$-hour recall.

levels higher than would be expected from their reported $24 \mathrm{HR}$ information. However, results were not changed substantively with removal of these participants, and a significant linear association between log-transformed urinary DTC levels and the $24 \mathrm{HR}$ data remained $(P<$ $0.02)$. Therefore, all data were retained for further analysis. Using the 24HR estimate of Cruciferae intake as a dependent variable in the linear regression model, each $\log$ unit change in DTC excretion $\left(\log \left(\mu \mathrm{mol} \mathrm{day}^{-1}\right)\right)$ was associated with approximately $35 \mathrm{~g} \mathrm{day}^{-1}$ of Cruciferous vegetable consumed.

In typical dietary investigations, the correlation between a detailed reference assessment (i.e. 24HR) and a summary assessment approach applicable for larger studies (e.g. urinary DTC, VFQ) would 'validate' the applied method, with recognition of the potential for correlated errors across the methods and a lack of a true gold standard. At Post-intervention, the correlation between urinary DTC and 24HR estimates was $0.57(P<0.05)$ (Table 2$)$. Using a structural equation approach, the data from the VFQ were incorporated into the evaluation of Cruciferous vegetable consumption and urinary DTC evaluation. The validity coefficient summarising the association between urinary DTC and the 'unknown but true intake' increased to 0.65. Adjusting the Pearson correlation coefficients for BMI, energy $\left(\mathrm{kcalday}^{-1}\right)$, social approval score or social desirability score had little affect. However, adjustment of Pearson correlation coefficients for the age of

Table 2 Correlation and validity coefficients for Cruciferae intake

\begin{tabular}{|c|c|c|}
\hline & $\begin{array}{l}\text { Baseline } \\
(n=27)\end{array}$ & $\begin{array}{l}\text { Post-intervention } \\
\quad(n=33)\end{array}$ \\
\hline \multicolumn{3}{|c|}{ Correlation coefficients $(95 \% \mathrm{Cl})$} \\
\hline$r_{\mathrm{VFQ} \times 24 \mathrm{HR}}$ & $0.56(0.22,0.77)$ & $0.67(0.43,0.82)$ \\
\hline$r_{\mathrm{VFQ} \times \mathrm{DTC}}$ & $0.22(-0.17,0.56)$ & $0.49(0.17,0.71)$ \\
\hline$r_{24 \mathrm{HR} \times \mathrm{DTC}}$ & $0.42(0.04,0.69)$ & $0.57(0.28,0.76)$ \\
\hline \multicolumn{3}{|c|}{ Validity coefficients $(95 \% \mathrm{Cl})$} \\
\hline VFQ & $0.54(0.18,0.87)$ & $0.76(0.47,0.92)$ \\
\hline DTC & $0.42(0.09,0.91)$ & $0.65(0.35,0.90)$ \\
\hline 24HR & $1.00(0.58,1.00)^{*}$ & $0.82(0.65,1.00) \dagger$ \\
\hline
\end{tabular}
as estimated by the vegetable and fruit questionnaire (VFQ), 24hour recall $(24 \mathrm{HR})$ and urinary dithiocarbamate excretion (DTC)

$95 \% \mathrm{Cl}-95 \%$ confidence interval.

*Validity coefficients and confidence intervals truncated at 1.00 .

† Upper limit of confidence interval truncated at 1.00 . 

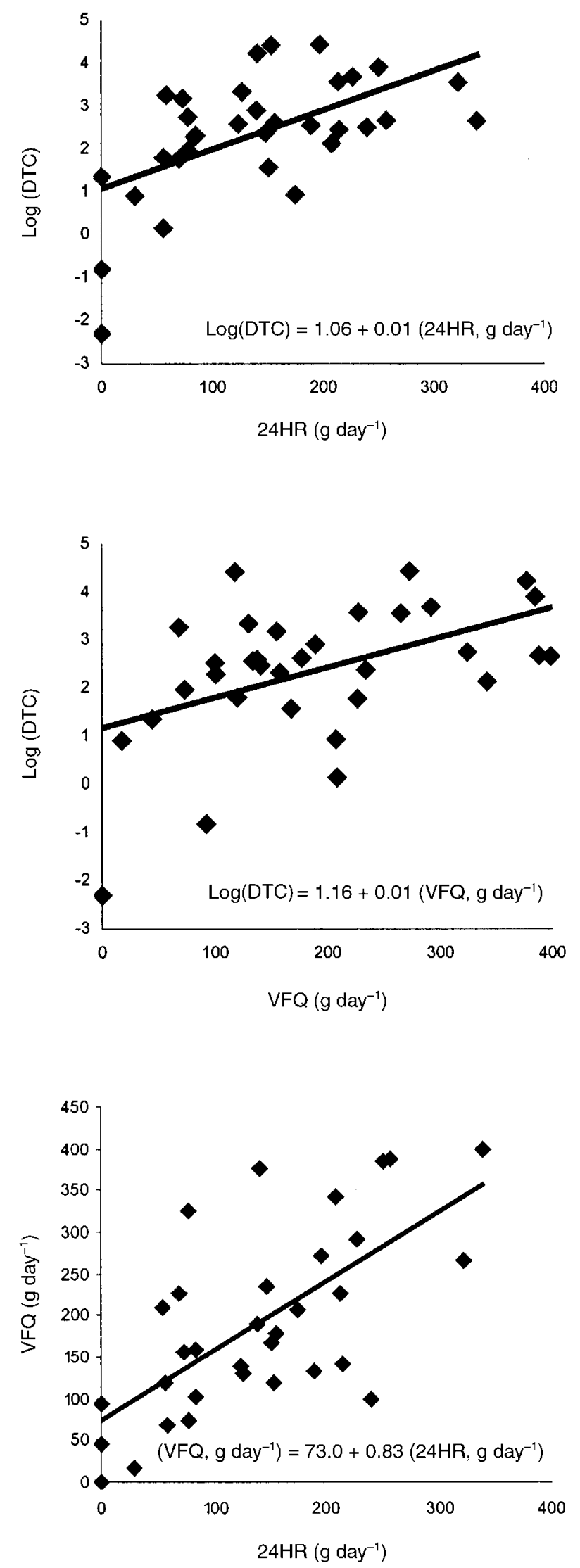

Fig. 1 Relationships between the three estimates of intake: 24hour recall (24HR), vegetable and fruit questionnaire (VFQ) and urinary dithiocarbamate excretion (DTC) - Post-intervention participants led to an increase in the urinary DTC validity coefficient from 0.65 to 0.77 (95\% CI $0.57,0.97$ ).

Baseline Cruciferae consumption was significantly lower than Post-intervention consumption (paired $t$-test: $P<0.001$ ), and most participants did not consume any of these vegetables during the reporting period (Table 1). There were no significant linear relationships between any of these dietary assessment methods (Fig. 2), illustrating the difficulty of measuring intake of a single food group within a low overall pattern of intake and wide availability of food choice. The validity coefficient for urinary DTC excretion was similar to the Pearson correlation between urinary DTC and the $24 \mathrm{HR}$, suggesting the structured equation approach provided no additional information when Cruciferae consumption is low. Furthermore, calculations for the $24 \mathrm{HR}$ validity coefficient produced a Heywood case, where the value of the validity coefficient was greater than 1.0 either due to chance or a violations of at least one analysis assumption.

\section{Discussion}

Standard dietary assessment approaches may be inadequate to evaluate the relationships between vegetable intake and disease risk. Urinary DTC excretion levels provide a food-specific biomarker for exposure to the glucosinolates found exclusively in Cruciferous vegetables. Prior to the intervention intake was sporadic, and the associations between the DTC biomarker and participant-reported data were unstable. However, with greater Cruciferae intake, we found urinary DTC levels were significantly correlated with both 24-HR and VFQ estimates. Since there is no 'gold standard' for dietary vegetable intake, we used a structural equation approach to estimate the relationship between the DTC biomarker and the study population's intake level. The association between DTC and intake increased $(r=0.65)$, providing further evidence that a single urinary DTC measurement would serve as a practical and reasonably accurate index of recent intake among a study population habitually consuming these vegetables.

The 24HR is an open-ended and interviewer-based referent dietary assessment approach often used to 'validate' other dietary assessment approaches, but is impractical for most large-scale epidemiological studies. We administered three $24 \mathrm{HRs}$ during a 7 -day period, an unusually high intensity of measurement, intended to provide a highly reliable approximation of each participant's intake during that 7-day period. For reasons related to random selection of intake days, the $24 \mathrm{HRs}$ were not consistently administered during the three days immediately prior to the day of urine collection, when dietary intake would be expected to have the greatest impact on urinary DTC excretion. The intake of specific foods, food groups and many micronutrients is more variable on a day-to-day basis than is daily intake of total energy or 

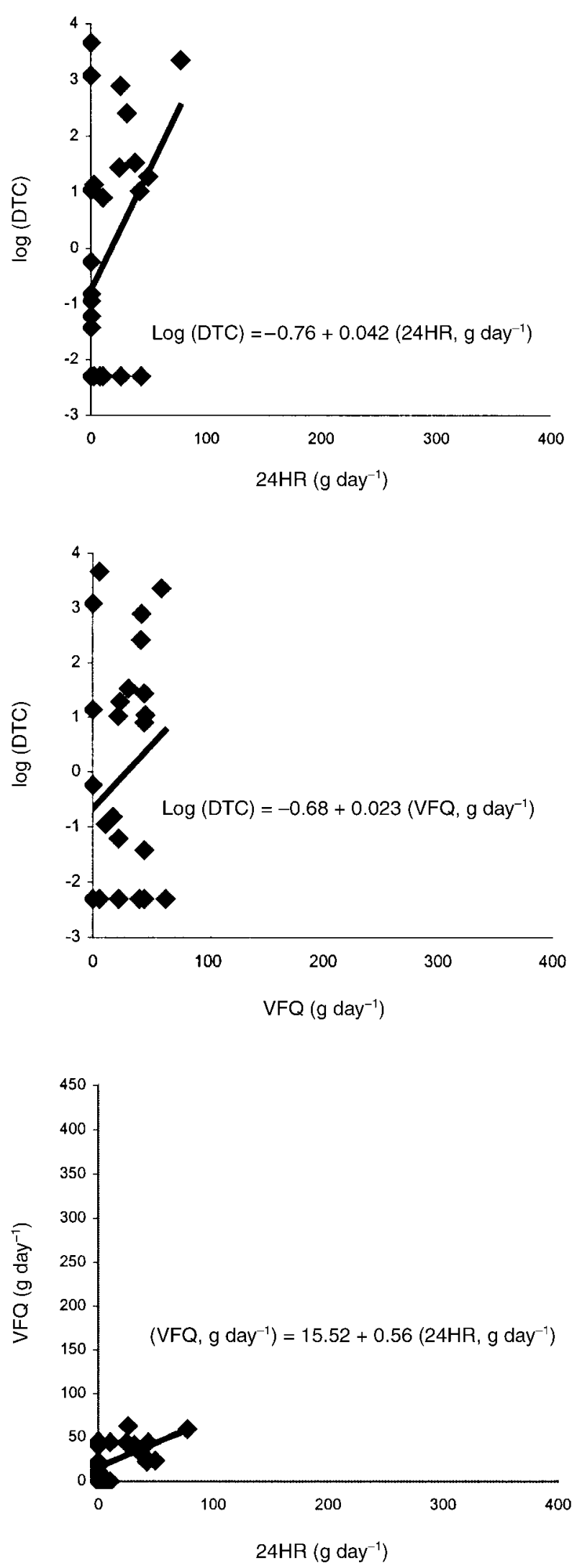

Fig. 2 Relationship between the three estimates of intake: 24hour recall (24HR), vegetable and fruit questionnaire (VFQ) and urinary dithiocarbamate excretion (DTC) - Baseline more commonly consumed nutrients ${ }^{21,22}$, resulting in higher within-person variability for foods such as Cruciferae as compared with macronutrients. Therefore, we administered the VFQ to complement the 24HR approach by measuring intake during the 7 days prior to urine collection. Procedural differences in administration, recall length and portion size estimation suggest that the dietary assessment measurement errors between the VFQ and 24HR are independent, but it is difficult to evaluate this assumption.

Kaaks and colleagues extended structural equation analyses to dietary assessment methods ${ }^{7,23}$. By combining three different dietary assessment approaches (Method of Triads), it may be possible to compensate for the lack of a dietary gold standard and estimate of the association between each measurement and the unknown 'true' intake. This approach should include a biological marker of dietary assessment, since dietary biomarker levels are unlikely to be associated with factors related to dietary misreport $^{7}$. At Post-intervention, the correlation between urinary DTC levels and 24HR estimates of intake was 0.57 , and the strength of this biomarker-24HR association compares favourably with other self-reported intake and biomarker associations reported previously ${ }^{24-27}$. Accommodation for potential measurement error in the $24 \mathrm{HR}$ assessment by inclusion of the VFQ evaluation produced a validity coefficient of 0.65 , indicating that the urinary biomarker should, at minimum, be sufficient to rank-order intake levels across subjects.

From controlled clinical studies, ITC is rapidly absorbed across a range of glucosinolate exposures, and the majority of DTC is excreted in urine within 8 hours of Cruciferae consumption ${ }^{6,10}$. We collected 24-hour urine specimens to capture this 8-hour window of time among free-living subjects, regardless of the time of day the vegetables were consumed. There was a weak but significant linear component between untransformed DTC excretion $\left(\mu \mathrm{mol} \mathrm{day}^{-1}\right)$ and dietary intake $(24 \mathrm{HR}$ $\left(\right.$ g day $\left.\left.^{-1}\right): \quad P=0.03, R^{2}=0.13\right)$; however, natural $\log$ transformation strengthened the observed linear fit $(P<$ $0.001, R^{2}=0.33$ ). Biomarker patterns often follow a lognormal distribution due to metabolic regulation of agent absorption, retention or excretion (e.g. GST activity). However, the absorption/excretion profile observed in previous clinical studies suggests that log transformation may also have reduced the influence of outliers, possibly due to dilution or degradation of DTC in the 24-hour urine collection or to variations in cooking practices (see below). This linear trend between dietary Cruciferae intake and urinary DTC levels provides confidence that urinary DTC levels are sufficient to rank-order Cruciferae consumption among subjects in an epidemiological investigation. We found each log unit of DTC excretion was associated with $35 \mathrm{~g} \mathrm{day}^{-1}$ of Cruciferous vegetable intake. However, our calibration between urinary DTC and Cruciferae intake should be repeated with attention to 
refrigeration during the 24-hour urine collection to eliminate the untested possibility of microbial contamination or DTC breakdown.

Cruciferae consumption levels exceeding those typically observed in the USA were necessary to detect an association between urinary DTC level and dietary Cruciferae intake. At Baseline, the DTC evaluation was affected by a few highly influential observations. Our study was limited in size, and therefore more easily influenced by chance outliers. Unusually high DTC levels at Baseline could result from ingestion of mustards, horseradishes and certain medications, or tobacco smoke exposure. Participants did not smoke or use prescription medications that might interfere with the cyclocondensation (DTC) assay, and all participants were instructed to avoid the consumption of condiments. However, we were unable to control for potential confounding due to ingestion of condiments or exposure to side-stream tobacco smoke. Such uncontrolled events may lead to the calculation of validity coefficients greater than 1.0 or less than -1.0 , the natural bounds of a correlation coefficient. We observed such a Heywood case in the validity coefficient for the $24 \mathrm{HR}$ at Baseline, but not at Post-intervention, where the higher intake levels would be expected to minimise the influence of these exposures. Additionally, the upper limits of our bootstrap confidence intervals may have exceeded 1.0 simply due to chance when repeatedly sampling from the study data. We truncated such values at 1.0 for easier presentation, with the understanding that our ability to conduct statistical testing and produce highly precise coefficients would be limited in this evaluation. Larger sample sizes conducive to more precise estimation may reduce the likelihood of chance occurrences and would provide the opportunity to investigate non-linear associations or alternative sources of bias in dietary reporting.

We explored the sensitivity of the validity coefficients to factors potentially affecting dietary reporting or urinary excretion levels. Partial correlation coefficients adjusted for energy intake $\left(\mathrm{kcalday}^{-1}\right)$, BMI $\left(\mathrm{kg} \mathrm{m}^{-2}\right)$, social desirability score or social approval score were similar to the crude correlation coefficients. We found that age (years) adjustment led to an increase in the correlation between $24 \mathrm{HR}$ and urinary DTC values, and increased the validity coefficient to 0.77 . We know no reason why age should affect urinary DTC excretion levels, nor do we know why age might affect dietary reporting via the $24 \mathrm{HR}$ approach but not the VFQ approach.

Vegetable cooking leads to a dramatic reduction in glucosinolate and ITC levels ${ }^{28-30}$. However, we found that the correlation between urinary DTC level and the amount of cooked Cruciferae consumed was similar to the correlation with the amount of raw Cruciferae consumed (not shown), suggesting that the value of the validity coefficients may be similar across food preparation patterns. Unfortunately, our VFQ assessment approach, as with most pencil-and-paper assessment methods, did not query for food preparation. Therefore, we were unable to calculate validity coefficients for urinary DTC excretion stratified by food preparation. The impact of food preparation on interpretation of DTC as a dietary biomarker may be less relevant in Asian populations, as traditional vegetable preparation approaches such as stirfrying provide little opportunity for glucosinolate loss. Our $24 \mathrm{HR}$ study data indicate that about $75 \%$ of vegetable servings were consumed after being cooked; however, our participants were provided practical skills in alternative cooking preparation techniques intended to prevent glucosinolate loss. Therefore, error in urinary DTC as a dietary biomarker may be minimal in Asian populations or special intervention groups, but modification of urinary DTC excretion levels by food preparation in a more general Western study population remains undetermined. Additionally, urinary DTC excretion may be modified by genetic polymorphisms in Phase 2 enzymes ${ }^{11}$ and degree of vegetable chewing, thus facilitating formation of ITC from glucosinolate precursors ${ }^{6}$. Further characterisation of cooking practices, eating habits, and Phase I and Phase II enzyme genetic profiles as possible determinants of DTC excretion should be considered for future investigations.

In summary, we continued our evaluation of urinary DTC excretion as a dietary biomarker for use in epidemiological investigations. We found urinary DTC levels strongly correlated with two alternative dietary assessment approaches. A structural equation evaluation suggests that urinary DTC levels present an accurate appraisal of a group's recent Cruciferous vegetable intake comparable to the $24 \mathrm{HR}$ or food-counting approaches. Future diet and cancer investigations should consider supplementing standard dietary assessment approaches with urinary DTC excretion measurement as a biomarker specific for Cruciferae intake.

\section{Acknowledgements}

This work was supported by a Biomedical Research Support Grant from the Office of Research (1998-9) and the Division of Preventive and Behavioral Medicine, University of Massachusetts Medical School; a gift from the Lewis B and Dorothy Cullman Foundation to the Brassica Chemoprotection Laboratory at Johns Hopkins University; the Department of Epidemiology and Biostatistics, University of South Carolina; and the Department of General and Internal Medicine and the Vanderbilt-Ingram Cancer Center, Vanderbilt University. Technical assistance was provided by Katherine K. Stephenson.

\section{References}

1 Michaud DS, Spiegelman D, Clinton SK, Rimm EB, Willett WC, Giovannucci EL. Fruit and vegetable intake and 
incidence of bladder cancer in a male prospective cohort J. Natl. Cancer Inst. 1999; 91: 605-13.

2 Verhoeven DT, Goldbohm RA, van Poppel G, Verhagen H, van den Brandt PA. Epidemiological studies of Brassica vegetables and cancer risk. Cancer Epidemiol. Biomark. Prev. 1996; 5: 733-48.

3 Lin HJ, Probst-Hensch NM, Louie AD, Kau IH, Witte JS, Ingles SA, Frankl HD, Lee ER, Haile RW. Glutathione transferase null geonotype, broccoli, and lower prevalence of colorectal adenomas. Cancer Epidemiol. Biomark. Prev. 1998; 7: 647-52.

4 Hebert JR, Peterson KE, Hurley TG, Stoddard AM, Cohen N, Field AE, Sorensen GT. The effect of social desirability trait on self-reported dietary measures among multi-ethnic female health center employees. Ann. Epidemiol. 2001; 11: 417-27.

5 Hebert JR, Clemow L, Pbert L, Ockene IS, Ockene JK. Social desirability bias in dietary self-report may compromise the validity of dietary intake measures. Int. J. Epidemiol. 1995; 24: 389-98.

6 Shapiro TA, Fahey JW, Wade KL, Stephenson KK, Talalay P. Chemoprotective glucosinolates and isothiocyanates of broccoli sprouts: metabolism and excretion in humans. Cancer Epidemiol. Biomark. Prev. 2001; 10: 501-8.

7 Kaaks RJ. Biochemical markers as additional measurements in studies of the accuracy of dietary questionnaire measurements: conceptual issues. Am. J. Clin. Nutr. 1997; 65: 1232-9.

8 Ye L, Dinkova-Kostova AT, Wade KL, Zhang Y, Shapiro T, Talalay P. Quantitative determination of dithiocarbamates in human plasma, serum erythrocytes, and urine: pharmacokinetics of broccoli sprout isothiocyanates in humans. Clin Chim. Acta 2002; 316: 43-53.

9 Zhang Y, Wade KL, Prestera T, Talalay P. Quantitative determination of isothiocyanates, dithiocarbamates, carbon disulfide, and related thiocarbonyl compounds by cyclocondensation of 1,2-benzenedithiol. Anal. Biochem. 1996; 239: $160-7$.

10 Shapiro TA, Fahey JW, Wade KL, Stephenson KK, Talalay P. Human metabolism and excretion of cancer chemoprotective glucosinolates and isothiocyanates of Cruciferous vegetables. Cancer Epidemiol. Biomark. Prev. 1998; 7 : 1091-100.

11 Seow A, Shi C-Y, Chung F-L, Jiao D, Hankin JH, Lee H-P, Coetzee GA, Yu MC. Urinary total isothiocyanate (ITC) in a population-based sample of middle-aged and older Chinese in Singapore: relationship with dietary total ITC and glutathione $S$-transferase M1/T1/P1 genotypes. Cancer Epidemiol. Biomark. Prev. 1998; 7: 775-81.

12 London SJ, Yuan J-M, Chung F-L, Gao Y-T, Coetzee GA, Ross RK, Yu MC. Isothiocyanates, glutathione S-transferase M1 and T1 polymorphisms, and lung-cancer risk: a prospective study of men in Shanghai, China. Lancet 2000; 356: 724-9.

13 Fowke JH, Longcope C, Hebert JR. Brassica vegetable consumption shifts estrogen metabolism in healthy postmenopausal women. Cancer Epidemiol. Biomark. Prev. 2000; 9: 773-9.

14 Nugon-Baudon L, Rabot S. Glucosinolates and glucosinolate derivatives: implications for protection against chemical carcinogenesis. Nutr. Res. Rev. 1994; 7: 205-31.

15 Hebert JR, Ockene IS, Hurley TG, Luippold R, Well AD,
Harmatz MG. Development and testing of a seven-day dietary recall. J. Clin. Epidemiol. 1997; 50: 925-37.

16 Buzzard IM, Faucett CL, Jeffrey RW, McBane L, McGovern P, Baxter JS, Shapiro AC, Blackburn GL, Chlebowski RT, Elashoff RM, Wynder EL. Monitoring dietary change in a lowfat diet intervention study: advantages of using 24-hour dietary recalls vs food records. J. Am. Diet. Assoc. 1996; 96: 574-9.

17 Marlowe D, Crowne D. Social desirability and responses to perceived situational demands. J. Consult. Clin. Psychol. 1961; 25: 109-15.

18 Martin HJ. A revised measure of approval motivation and its relationship to social desirability. J. Pers. Assess. 1984; 48: 508-16.

19 Kleinbaum DG, Kupper LL, Muller KE. Applied Regression Analysis and Other Multivariable Methods. Boston, MA PWS-Kent Publishing, 1988.

20 Ocké MC, Kaaks RJ. Biochemical markers of additional measurements in dietary validity studies: application of the method of triads with examples from the European Prospective Investigation into Cancer and Nutrition. Am. J. Clin. Nutr. 1997; 65: 1240-5.

21 Decarli A, Franceschi S, Ferraroni M, Gnagnarella P, Parpinel MT, LaVecchia C, Negri E, Salvini S, Falcini F, Giacosa A. Validation of a food-frequency questionnaire to assess dietary intakes in cancer studies in Italy: results for specific nutrients. Ann. Epidemiol. 1996; 6: 110-8.

22 Salvini S, Hunter DJ, Sampson L, Stempfer M, Colditz GA, Rosner B, Willett WC. Food-based validation of a dietary questionnaire: the effects of week-to-week variation in food consumption. Int. J. Epidemiol. 1989; 18: 858-67.

23 Kaaks R, Riboli E, Estève J. Estimating the accuracy of dietary questionnaire assessments: validation in terms of structural equation models. Stats. Med. 1994; 13: 127-42.

24 Marshall JR, Lanza E, Bloch A, Caan G, Caggiula A, Quandt S, Iber F, Kikendall W, Slattery M, Sowell A. Indexes of food and nutrient intakes as predictors of serum concentrations of nutrients: the problem of inadequate discriminant validity. Am. J. Clin. Nutr. 1997; 65: 1269-74.

25 Ocke MC, Bueno-de-Mesquita HB, Pols MA, Smit HA, van Staveren WA, Kranhout D. The Dutch EPIC food frequency questionnaire. II. Relative validity and reproducibility for nutrients. Int. J. Epidemiol. 1997; 26: S49-58.

26 Willett W. Nutritional Epidemiology. New York: Oxford University Press, 1990.

27 Katsouyanni K, Rimm EB, Gnardellis C, Trichopoulos D, Polychronopoulos E, Trichopoulou A. Reproducibility and relative validity of an extensive semi-quantitative food frequency questionnaire using dietary records and biochemical markers among Greek schoolteachers. Int J. Epidemiol. 1997; 26: S118-27.

28 Howard LA, Jeffrey EH, Wallig MA, Klein BP. Retention of phytochemicals in fresh and processed broccoli. J. Food Sci. 1997; 62: 1098-100.

29 Fowke JH, Fahey JW, Stephenson K, Hebert JR. Using isothiocyanate excretion as a biological marker of Brassica consumption: evaluating the sources of variability. Public Health Nutr. 2001; 4: 837-46.

30 Fahey JW, Stephenson KK. Cancer chemoprotective effects of cruciferous vegetables. Horticult. Sci. 1999; 34: 1159-63. 


\section{Appendix - Vegetable and Fruit Questionnaire}

Please tell us how often you have eaten the specified food item, and the typical portion size in the past seven days, excluding today. All portion sizes refer to cooked size unless otherwise noted. Please write in the number of times that you have consumed the food and check off your usual portion size as compared to the Comparison Portion Size. For example, if you ate broccoli three times (one cup at one sitting and 1/4 cup the other two times).

\begin{tabular}{|c|c|c|c|c|c|}
\hline Broccoli & $\begin{array}{c}\text { Number of } \\
\text { times eaten } \\
3\end{array}$ & $\begin{array}{l}\text { Comparison portion size } \\
\qquad 1 / 2 \text { cup }\end{array}$ & Half this size & $\begin{array}{c}\text { Your average } \\
\text { equal to this size } \\
x\end{array}$ & Twice this size \\
\hline & $\begin{array}{l}\text { Number of } \\
\text { times eaten }\end{array}$ & Comparison portion size & Half this size & $\begin{array}{l}\text { Your average } \\
\text { equal to this size }\end{array}$ & Twice this size \\
\hline Broccoli & & $1 / 2$ cup & & & \\
\hline $\begin{array}{l}\text { Brussels sprouts } \\
\text { Cabbage }\end{array}$ & & $\begin{array}{l}4 \text { sprouts } \\
1 / 2 \text { cup }\end{array}$ & & & \\
\hline Cauliflower & & $1 / 2$ cup & & & \\
\hline Chinese cabbage & - & $1 / 2$ cup & & & \\
\hline $\begin{array}{l}\text { Collard greens/Swiss } \\
\text { chard/kohlrabi }\end{array}$ & & $1 / 2$ cup & & & \\
\hline $\begin{array}{l}\text { Mustard greens or turnip } \\
\text { greens }\end{array}$ & & $1 / 2$ cup & & & \\
\hline Rutabaga/turnips & & $1 / 2$ cup & & & \\
\hline Kale & & $1 / 2$ cup & & & \\
\hline Spinach & & $1 / 2$ cup & & & \\
\hline Onions & & 1 small or $1 / 4$ cup & & & \\
\hline Carrots & & 1 medium or $1 / 2$ cup & & & \\
\hline Sweet potatoes & & $1 / 2$ cup & & & \\
\hline Soybeans - whole & & $3 / 4$ cup & & & \\
\hline Soy milk - 8 oz glass & & $1 \times 8$ oz glass & & & \\
\hline Tofu & & $3 / 4$ cup & & & \\
\hline Tempeh & & $1 / 2$ cup & & & \\
\hline Broccoli sprouts & & $1 / 2$ cup & & & \\
\hline $\begin{array}{l}\text { Alfalfa/clover/mung bean/soy } \\
\text { sprouts (raw) }\end{array}$ & & $1 / 2$ cup & & & \\
\hline $\begin{array}{l}\text { Pinto beans/round split pea } \\
\text { pods }\end{array}$ & & $1 / 2$ cup & & & \\
\hline Fresh green or mung beans & & $1 / 2$ cup & & & \\
\hline $\begin{array}{l}\text { Garbanzo, kidney beans or } \\
\text { black-eyed, yellow split or } \\
\text { Chinese peas }\end{array}$ & & 1/2 cup & & & \\
\hline Peas & & $1 / 2$ cup & & & \\
\hline Lentils/dal & - & $\begin{array}{c}1 / 2 \text { cup } \\
1 / 4 \text { cot }\end{array}$ & & & \\
\hline $\begin{array}{l}\text { Seaweeds eaten dry (e.g. } \\
\text { dulse, purple laver, nori) }\end{array}$ & & $1 / 4$ cup or $7 "$ sq. sheet & & & \\
\hline $\begin{array}{l}\text { Seaweeds eaten cooked or } \\
\text { soaked (e.g. arame, kombu, } \\
\text { kelp) }\end{array}$ & & 1 tbs or $2^{\prime \prime}$ sq. piece & & & \\
\hline $\begin{array}{l}\text { Apples } \\
\text { Bananas }\end{array}$ & $\ldots$ & 1 medium or $1 / 2$ cup & & & \\
\hline $\begin{array}{l}\text { Bananas } \\
\text { Apricots }\end{array}$ & & 2 medium & & & \\
\hline Nectarines & 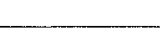 & 1 medium or $1 / 2$ cup & & & \\
\hline Peaches & & 1 medium or $1 / 2$ cup & & & \\
\hline Strawberries & & $1 / 2$ cup & & & \\
\hline Grapefruit & & $1 / 2$ grapefruit & & & \\
\hline $\begin{array}{l}\text { Lemon, squeezed } \\
\text { Orange }\end{array}$ & & $\begin{array}{l}1 / 4 \text { medium } \\
1 \text { medium }\end{array}$ & & & \\
\hline $100 \%$ Fruit juice (any type) & & $1 \times 8$ oz glass & & & \\
\hline \multicolumn{6}{|l|}{$\begin{array}{l}\text { Other soy products not listed } \\
\text { above }\end{array}$} \\
\hline Please speciry: & & your portion size. & & & \\
\hline
\end{tabular}

